\title{
Measuring Lifetime Poverty
}

\author{
Michael Hoy \\ University of Guelph \\ and \\ Buhong Zheng \\ University of Colorado Denver \\ Department of Economics, University of Guelph \\ Discussion Paper No. 2008-14 \\ December, 2008 \\ Abstract: This paper presents an axiomatic framework for measuring life- \\ time poverty over multiple periods. For an individual, we argue that \\ lifetime poverty is influenced by both the "snapshot" poverty of each \\ period and the poverty level of the "permanent" lifetime consumption; \\ it is also influenced by how poverty spells are distributed over the life- \\ time. Two obvious candidates for aggregation are to aggregate over time \\ and then across individuals, or vice versa. For a society, we consider a \\ path-independence requirement to make these two approaches of aggrega- \\ tion consistent. We axiomatically characterize classes of lifetime poverty \\ indices and derive dominance conditions of poverty orderings for both
} individual and societal lifetime poverty measurements.

JEL Classifications: I32

Key Words: Lifetime poverty, snapshot poverty, chronic poverty, poverty measurement.

Acknowledgement: For very helpful comments we thank Walter Bossert, Conchita D'Ambrosio, Serge Kolm and other participants of our presentation at the 8th International Meeting of the Society for Social Choice and Welfare (July 2006, Istanbul). The first author thanks SSHRC for financial support. 


\section{Measuring Lifetime Poverty}

\section{Introduction}

Suppose two individuals live through the same number of periods of time and both are poor during some of the periods. Under what circumstances can we say that one person is poorer than the other from the perspective of their lifetime poverty experience? The conventional literature on poverty measurement is of little help in answering the question in a general way except perhaps in the case where one of the individuals has more income than the other for all periods in which one or the other experiences poverty. In this case, it may seem reasonable to conclude that the person with more income has less lifetime poverty than the other person. But as long as the two individuals' poverty spells are different (i.e., one person lives in poverty while at the same time the other lives out of poverty) or neither individual has more income for all of the poverty periods, little can be said about lifetime poverty comparison since the comparison requires the evaluation of poverty over time - and there are no axioms in the conventional literature to enable that. To expand the literature of poverty measurement to lifetime poverty, therefore, it is necessary to investigate the notion of lifetime poverty for an individual as well as for a society, and to identify the appropriate axioms for their measurement.

This paper provides such an axiomatic framework. The measurement for individual lifetime poverty consists of two steps: the measurement of each individual's "snapshot poverty" at each period and the aggregation of these snapshot poverty spells across all periods. Here a period is interpreted as the basic unit of time that poverty is measured; income is collected at the beginning of each period to enable consumption in that period and in the subsequent periods. A person is poor in a period if and only if his consumption level in that period falls short of the poverty line. For the measurement of lifetime poverty, it is important to stress the use of consumption rather than disposable income in the calculation of poverty as consumption is a much more accurate measure of the standard of living over time. The measurement of snapshot poverty at each period is straightforward; each individual's poverty is measured as his consumption deprivation from the poverty line. The conventional literature on poverty measurement provides ample guidelines for this stage of the measurement.

It is the second stage of lifetime poverty measurement that expands the literature on poverty measurement. When viewed from a lifetime perspective, the suffering and deprivation of each individual in each period transmits into the lifetime evaluation of poverty. All other things equal, the more deprivation a person endures in a given period, the more lifetime poverty is created for or experienced by the individual. This "experience axiom" is akin to the monotonicity axiom or the subgroup consistency axiom typically used in the measurement of snapshot poverty for a single period of time. All these snapshot poverty experiences considered in isolation of each other, however, may not suffice to determine lifetime poverty. One should also account for 
periods in which an individual lives out of poverty. Over a lifetime an individual, in retrospect, may well benefit from high consumption experienced in non-poverty spells. Given that the deprivation in one period can be offset at least in part by the experience of affluent living in another period, lifetime poverty is also influenced by consumption over one's lifetime as a whole when it is compared with a sort of "lifetime poverty line." The essence of this argument is reflected in our "retrospective axiom" which stipulates that lifetime poverty is a function of the lifetime permanent consumption poverty (in the paper the permanent consumption is approximated by the average lifetime consumption in the absence of discounting). This reflects a sense of how an individual has experienced lifetime poverty from a retrospective view of his/her consumption profile. The particular pattern or timing of poverty spells also may affect the overall lifetime poverty of an individual. We explore further axioms on the timing of poverty spells (see below), along with other regularity assumptions, to derive a general class of lifetime poverty indices that are the weighted sum between the average snapshot poverty level across all periods and the poverty of average lifetime consumption.

To further characterize the class of lifetime poverty indices, we propose two additional axioms governing the aggregation of snapshot poverty across time. These axioms are sensitive to the particular pattern of poverty spells over time. The first of these we call the early poverty axiom. There is substantial evidence that poverty in earlier stages of life not only affects consumption in later periods but also leaves an inherently deeper mark on lifetime deprivation. Recent research in neuroscience (e.g., see Farah, et al., 2006), suggests that children growing up in poor families with low social status not only suffer from inadequate nutrition and exposure to environmental toxins but also suffer from elevated stress hormones that generally impair neural development, including effects on language and memory. ${ }^{1}$ This suggests two channels for adverse effects on the individual later in life. First is a direct impact of poverty early in life in that, due to the vulnerability of children, the physiological and psychological effects noted above reduce their future enjoyment from life for any given future (continuing) stream of consumption levels. Secondly, early poverty worsens an individual's capability to generate higher consumption later in life due to a compromised ability to accumulate human capital and obtain favorable employment opportunities. This second effect is captured implicitly by the fact that we include all future levels of consumption in our measurement of lifetime poverty. However, to account for the first (direct) effect, we require greater weight to be placed on poverty experienced earlier in life. Note that if, alternatively, one feels that poverty at both early and late life should be weighted more - say due to the special vulnerability of both the young and old to spells of poverty - then the weighting function should be U-shaped. It would, of course, be possible to adopt such an axiom within our

\footnotetext{
${ }^{1}$ See also the reports on this line of research from the 2008 meetings of the American Association for the Advancement of Science (Boston) described in an article by Clive Cookson (The Financial Times, February 16, 2008) and also by Paul Krugman (New York Times, February 18, 2008).
} 
framework and analyze its impact in much the same way as we analyze the impact of our early poverty axiom.

There is a recognition that repeated years or spells of poverty are particularly harmful to individual well-being. This is reflected in empirical papers that attempt to distinguish between transient and chronic poverty and their causes and the development of policies specifically directed at reducing long-term poverty experiences of individuals (e.g., see Jalan and Ravallion (1998, 2000), Baulch and Hoddinott (2000), and Duclos, Araar, and Giles (2006)). We introduce an axiom, the "chronic poverty axiom" that incorporates this concern. The axiom implies that multiple poverty spells experienced concurrently or separated by only a few periods of nonpoverty are more harmful to an individual's well-being than would be the case if the same set of 'snapshot poverty experiences' were more spread out over time. The implication of our chronic poverty axiom is that the weighting function used to aggregate snapshot poverty experiences is concave in the time period.

As Addison, Hulme, and Kanbur (2008, p. 8) note, however: "The introduction of time into the economic theory of poverty measurement is relatively recent." Only a few other recent papers have focussed on developing a measurement framework to reflect chronic poverty. One principal contribution of our paper is to integrate individuals' chronic poverty experiences into a framework of lifetime poverty measurement using a formal axiomatic approach. We discuss how our approach differs from these other recent contributions in the concluding section.

Conceptually, the measurement of lifetime poverty for a society can have two alternative approaches to follow: (1) measuring each individual's lifetime poverty first and then aggregating across all individuals in the society; and (2) measuring society's aggregate poverty in each period and then aggregating them across time in a manner similar to the measurement of individual lifetime poverty. These two approaches represent two different paths to obtain the same goal of obtaining a societal lifetime poverty index. It is useful to consider the situation where the two approaches yield the same result. This path-independence consideration leads to a specific functional form for the societal lifetime poverty index. When all path-independent lifetime poverty indices are considered, a set of poverty ordering conditions are also derived. In these conditions, income mobility - income movement among people over time - is shown to play a role in determining a society's lifetime poverty; all other things equal, a more mobile society tends to have less aggregate lifetime poverty.

The approach of our paper is related to the seminal paper by Rodgers and Rodgers (1993). Their primary goal is to distinguish between chronic (persistent) and transitory poverty while ours is to more fully exploit the richness in the pattern of poverty spells using axioms that are sensitive to both a concern with early and chronic poverty experiences. To measure the aggregate poverty of society, they use the weighted average snapshot poverty of the society across the time periods considered; the weight being the proportion of the population present in each time period. As we have explained above, we believe this simple averaging process is far from adequate in 
measuring the lifetime poverty of a society. Our paper is also related to the two papers by Karcher, Moyes and Trannoy (1995, 2002) where they measure society's aggregate social welfare over time with different discounting concerns. Unlike their characterizations, our approach does not rely on a concern with discounting and is axiomatically characterized. Also income/consumption mobility plays a role in our measurement/rankings of lifetime poverty.

The rest of the paper is organized as follows. The next section characterizes individual lifetime poverty. Here we propose a set of axioms that are pertinent to measuring poverty over time. When all members of the class are considered for poverty orderings, we derive the corresponding dominance conditions that are related to the familiar "concentration dominances" for various sets of axioms. We also illustrate the strength of the resulting orderings using pairwise comparisons of income profiles from the PSID data set. Section III considers the aggregation of individual lifetime poverty across society. Section IV provides some additional remarks and also concludes the paper.

\section{The Measurement of Individual Lifetime Poverty}

Consider an individual who lives through $T$ periods. Each period can be interpreted as a year or as a phase of life such as youth, middle age and old age. In each period $t, t=1,2, \ldots, T$, the individual has a non-negative level of consumption $x_{t}$. In each period, the individual's poverty status is determined by comparing his consumption level with the poverty line $0<z<\infty$ which is exogenously given and remains constant throughout the $T$ periods. The individual is poor in period $t$ if and only if his consumption level $x_{t}$ is strictly less than $z$. Denote $\mathbf{x}=\left(x_{1}, x_{2}, \ldots, x_{T}\right)^{\prime}$ the profile of the individual's lifetime consumptions, his lifetime poverty level is a function $P(\mathbf{x} ; z)$ which maps each consumption profile $\mathbf{x}$ into $[0, \infty)$. The average consumption of the individual over the $T$ periods is $\bar{x}$. For each consumption variable, we also define its censored consumption as $\tilde{x}_{t}=\min \left\{x_{t}, z\right\}$. In what follows, we discuss appropriate axioms that can be imposed upon the functional form of $P(\mathbf{x} ; z)$.

At the beginning of each period, the individual collects income and allocates it to the consumptions of that period and the periods to come; at the end of each period, the individual compares his consumption level $x_{t}$ with the poverty line $z$. If $x_{t}<z$, he has poverty deprivation which is measured by $p\left(x_{t} ; z\right): p\left(x_{t} ; z\right)>0$ if $x_{t}<z$; otherwise he lives out of poverty: $p\left(x_{t} ; z\right)=0$ if $x_{t} \geq z$. We refer to $p\left(x_{t} ; z\right)$ as the "snapshot poverty" of period $t$. The measurement of poverty deprivation has been well studied in the literature. In general, we assume that $p\left(x_{t} ; z\right)$ is continuous, $\frac{\partial p\left(x_{t} ; z\right)}{\partial x_{t}}<0$ and $\frac{\partial^{2} p\left(x_{t} ; z\right)}{\partial x_{t}^{2}}>0$ for all $x_{t} \in[0, z){ }^{2}$ That is, poverty deprivation decreases as consumption increases; it decreases, however, at a slower pace as consumption increases - in part

\footnotetext{
${ }^{2}$ Other axioms in the literature such as the increasing poverty line axiom $-p\left(x_{t} ; z\right)$ is increasing in $z$ - and the unit-consistency axiom (Zheng, 2007) - which implies that $p\left(x_{t} ; z\right)$ is a homogeneous function of $x_{t}$ and $z$ - may also be considered to further specify the functional form of $p\left(x_{t} ; z\right)$.
} 
this is to reflect the poverty aversion consideration (Zheng, 2000). Although higherorder conditions can be entertained, in this paper we limit our investigation to only the first two orders. ${ }^{3}$ Accordingly, we also assume the lifetime poverty measure $P(\mathbf{x} ; z)$ to exhibit similar properties as $p(x ; z)$, i.e., $\frac{\partial P(\mathbf{x} ; z)}{\partial x_{t}} \leq 0$ and $\frac{\partial^{2} P(\mathbf{x} ; z)}{\partial x_{t}^{2}} \geq 0$ for all $x_{t} \in[0, \infty)$. Note that here we require only weak inequalities and the range for $x_{t}$ is over $[0, \infty)$ rather than $[0, z)$. This is because, unlike in the measurement of snapshot poverty where any change in $x_{t}$ above $z$ has no effect on the poverty level, here such a change may or may not affect the lifetime poverty - as we will see below.

Our first axiom establishes the connection between the "snapshot poverty" of each period $p\left(x_{t} ; z\right)$ and that of lifetime $P(\mathbf{x} ; z)$.

The experience axiom: $P(\mathbf{x} ; z)$ is an increasing function of $p\left(x_{t} ; z\right)$ for $t=1,2, \ldots, T$.

What this axiom states is pretty clear: the suffering from poverty deprivation in each period is transmitted directly and positively into the lifetime poverty deprivation. Recounting at the end of the $T$ th period, the individual may feel life has been harder if he had a poor childhood than otherwise - all else the same. This is certainly a reasonable requirement: if $p\left(x_{t} ; z\right)$ increases then it must be $x_{t}<z$ and $x_{t}$ decreases which, in turn, must increase the lifetime poverty deprivation. This interpretation is the usual monotonicity axiom used in the snapshot poverty measurement applied to the lifetime context. This axiom is also akin to the familiar subgroup consistency axiom which says that if a subgroup experiences an increase in poverty, then the overall society's poverty ought to go up. Viewing the individual's consumption in each year as a subgroup and the lifetime consumption profile as a society, then the experience axiom becomes analogous to the subgroup consistency axiom.

But the individual's picture of lifelong living is not entirely dictated by the poverty spells that he has experienced in the various periods. He might view that "even though I had a rough childhood, life as a whole has been very good to me since I had an affluent living later in my life." This means that the individual registers all poverty deprivations but we also allow periods of rich living in the rest of his lifetime to offset some of the bad experiences or memories. How to capture this offsetting phenomenon? A natural and manageable way to consider poverty for the entire lifetime in a retrospective manner is to model consumption over the lifetime as if it were completely smoothed out. The poverty level is then computed by comparing lifetime (permanent) consumption with the lifetime (permanent) poverty line. Since we assume the poverty line remains the same throughout all $T$ periods, all consumption levels will not be discounted either (or consider they are already discounted). It follows that we can proxy permanent consumption with a simple average consumption

\footnotetext{
${ }^{3}$ Our choice also reflects the fact that poverty orderings at third and above orders may collapse to second-order if the poverty line is uncertain and expands over a large interval (Zheng, 1999). With uncertain poverty lines, the consideration of higher-than-second-order conditions may introduce little additional insights on poverty orderings. Shorrocks and Foster (1987) and Davies and Hoy (1994, 1995) explore the implications of third-order stochastic dominance in making inequality comparisons.
} 
over the lifetime. ${ }^{4}$ Noting that $p(\bar{x} ; z)$ is the poverty of lifetime average consumption, our second axiom summarizes the afore-discussed influence of lifetime smoothed consumption on lifetime poverty.

The retrospective axiom: $P(\mathbf{x} ; z)$ is an increasing function of $p(\bar{x} ; z)$.

With the retrospective axiom, the focus axiom in the literature (i.e., that any change made in any above-the-poverty-line consumption has no affect on the poverty level) needs to be modified. Since now a change in a consumption may affect lifetime poverty through two routes: through the snapshot poverty in each period and through the poverty of average-lifetime-consumption. It follows that the new focus axiom should be reformulated as: a change in a period's consumption has no effect on lifetime poverty if and only if both the consumption level in that period and the average-consumption level of the entire lifetime are above the poverty line. This is to say $\frac{\partial P(\mathbf{x} ; z)}{\partial x_{t}}<0$ if either $x_{t}<z$ or $\bar{x}<z$; otherwise $\frac{\partial P(\mathbf{x} ; z)}{\partial x_{t}}=0$ - as we have assumed at the beginning of this section.

The experience axiom and the lifetime axiom together imply that the lifetime poverty $P(\mathbf{x} ; z)$ is an increasing function of $p\left(x_{t} ; z\right)$ for $t=1,2, . ., T$ and $p(\bar{x} ; z)$. To fully calibrate the relation among the three types of poverty, a stronger axiom than both the experience axiom and the lifetime axiom is needed.

The strong monotonicity axiom: for two consumption profiles $\mathbf{x}$ and $\mathbf{y}, P(\mathbf{x} ; z)>$ $P(\mathbf{y} ; z)$ if $p\left(x_{t} ; z\right) \geq p\left(y_{t} ; z\right)$ for $t=1,2, . ., T$ and $p(\bar{x} ; z) \geq p(\bar{y} ; z)$ with at least one inequality holding strictly.

The strong monotonicity axiom implies that the lifetime poverty is uniquely determined by $\left\{p\left(x_{t} ; z\right)\right\}$ and $p(\bar{x} ; z)$. Clearly, the strong monotonicity axiom implies both of the previous axioms but it is not implied by either one of them or both of them. To see how the conjunction of snapshot poverty and lifetime poverty operate differently from consideration of one on its own, consider the following example.

Example: Let $z=5$ and $T=3$. We have for $\mathbf{x}=(1,3,7)$ and $\mathbf{y}=(2,4,8)$ both that $p\left(x_{t} ; z\right) \geq p\left(y_{t} ; z\right)$ for all $t$ (with strict inequality for some $t$ ), and $p(\bar{x} ; z) \geq p(\bar{y} ; z)$ since $\bar{x} \leq \bar{y}$. Moreover, in this case we have $\bar{x}<\bar{y}<z$ which implies $p(\bar{x} ; z)>$ $p(\bar{y} ; z)$. Thus, even though we don't have $x_{r} \leq y_{s}, \forall r, s \in\{1,2,3\}$, the evaluation of snapshot poverty and retrospective poverty (lifetime average consumption poverty) operate in the same direction and we have $P(\mathbf{x} ; z)>P(\mathbf{y} ; z)$ according to the strong monotonicity axiom in conjunction with the lifetime and experience axioms. However, if we consider $\mathbf{x}^{\prime}=(1,3,14)$ we have that in considering snapshot poverty $\mathbf{x}^{\prime}$ displays more poverty than $\mathbf{y}$ but the opposite applies in comparing retrospective poverty. Thus, in this case the evaluation of snapshot and retrospective poverty do not agree and we cannot rank lifetime poverty from these two distributions.

\footnotetext{
${ }^{4}$ If a more suitable representation of permanent consumption is deemed necessary, we can replace $\bar{x}$ with such a permanent-consumption function $\mu\left(x_{1}, x_{2}, \ldots x_{T}\right)$ in the rest of the paper. All results involving $\bar{x}$ will also hold with some appropriate modifications. For example, one condition at the end of the next section $-F\left(x_{1}, \ldots, x_{t}, \ldots x_{T}\right) \geq G\left(x_{1}, \ldots, x_{t}, \ldots x_{T}\right)$ for all $x_{1}, \ldots, x_{t}, \ldots x_{T}$ such that $\bar{x}<z-$ becomes $F\left(x_{1}, \ldots, x_{t}, \ldots x_{T}\right) \geq G\left(x_{1}, \ldots, x_{t}, \ldots x_{T}\right)$ for all $x_{1}, \ldots, x_{t}, \ldots x_{T}$ such that $\mu\left(x_{1}, x_{2}, \ldots x_{T}\right)<z$.
} 
But in what way may these terms jointly determine the lifetime poverty? For each period's snapshot poverty $p\left(x_{t} ; z\right)$, we assume that its effect on the lifetime poverty $P(\mathbf{x} ; z)$ is independent of any other period's snapshot poverty $p\left(x_{s} ; z\right)$ for $s \neq t$ and of the poverty level of the average lifetime consumption $p(\bar{x} ; z)$. This axiom is akin to the decomposability axiom used in the snapshot poverty measurement; it enables researchers to compute the contribution of each year's consumption and the smoothed consumption to total poverty and allows policy-makers to identify the specific factors that are responsible for changes in the overall poverty value. This requirement amounts to saying that there is no interaction among the poverty levels of the various periods and that of the average consumption. Formally, this assumption can be stated as follows.

The independence axiom: $\frac{\partial^{2} P(\mathbf{x} ; z)}{\partial p\left(x_{t} ; z\right) \partial p\left(x_{s} ; z\right)}=0$ for all $s \neq t$ and $\frac{\partial^{2} P(\mathbf{x} ; z)}{\partial p\left(x_{t} ; z\right) \partial p(\bar{x} ; z)}=0$ for all $t=1,2, \ldots, T$.

Example: To illustrate the implications of the independence axiom, consider the following consumption vectors; $\mathbf{x}=(1,1,4,12), \mathbf{y}=(2,1,2,13)$ and $\mathbf{x}^{\prime}=(1,1+$ $\delta, 4,12), \mathbf{y}^{\prime}=(2,1+\delta, 2,13), \forall \delta \in[-1, M)$ for any finite $M>0$. The independence axiom implies that if $P(\mathbf{x} ; z)=P(\mathbf{y} ; z)$ then $P\left(\mathbf{x}^{\prime} ; z\right)=P\left(\mathbf{y}^{\prime} ; z\right)$. The reason is that altering income in period $t=2$, which is equal for the two distributions, in the same way has the same effect on snapshot poverty. Moreover, $\bar{x}=\bar{y}$ and so the change in income applied to obtain $\mathbf{x}^{\prime}$ and $\mathbf{y}^{\prime}$ also has the same effect on lifetime poverty. The independence axiom means that these are the only two implications of this change in consumption on $P(\mathbf{x} ; z)$ and $P(\mathbf{y} ; z)$ and so the resulting poverty levels for $\mathbf{x}^{\prime}$ and $\mathbf{y}^{\prime}$ must also be equal. Without the independence axiom, these (equivalent) changes in $p\left(x_{2} ; z\right)$ and $p\left(y_{2} ; z\right)$ could have changed snapshot poverty in any of the other periods $t=1,3$ differently for the two distributions and this would imply possibly different poverty levels for distributions $\mathbf{x}^{\prime}$ and $\mathbf{y}^{\prime}$. A similar argument could apply if the means of lifetime consumptions were different.

If an individual's consumption level is equal in all periods, i.e., $x_{s}=x_{t}$ for all $s$ and $t$, then it is reasonable to say that this individual's lifetime poverty can be appropriately represented by the snapshot poverty $p\left(x_{t} ; z\right)$. This axiom is similar to the normalization axiom used in the measurement of income inequality.

The normalization axiom: $P(\mathbf{x} ; z)=p(x ; z)$ if $x=x_{1}=x_{2}=\ldots=x_{T}$.

The following proposition documents the implication of the above three axioms for the functional form of $P(\mathbf{x} ; z)$.

Proposition 2.1. A lifetime poverty measure $P(\mathbf{x} ; z)$ satisfies the strong monotonicity axiom, the independence axiom and the normalization axiom if and only if there exist continuous and positive functions $f_{1}, f_{2}, \ldots, f_{T}$ and $g$ such that

$$
P(\mathbf{x} ; z)=\sum_{t=1}^{T} f_{t}(t) p\left(x_{t} ; z\right)+g(T) p(\bar{x} ; z)
$$

where $\sum_{t=1}^{T} f_{t}(t)+g(T)=1$. 
Proof. The strong monotonicity axiom implies that $P(\mathbf{x} ; z)$ is an increasing function of only $\left\{p\left(x_{t} ; z\right)\right\}$ and $p(\bar{x} ; z)$. That is, there exists a continuous and increasing function $f$ such that

$$
P(\mathbf{x} ; z)=f\left[p\left(x_{1} ; z\right), p\left(x_{2} ; z\right), \ldots, p\left(x_{T} ; z\right), p(\bar{x} ; z)\right] .
$$

The independence axiom implies that $P(\mathbf{x} ; z)$ is additively separable in $\left\{p\left(x_{t} ; z\right)\right\}$ and $p(\bar{x} ; z)$, i.e.,

$$
P(\mathbf{x} ; z)=\tilde{f}_{1}\left[p\left(x_{1} ; z\right)\right]+\tilde{f}_{2}\left[p\left(x_{2} ; z\right)\right]+\ldots \tilde{f}_{T}\left[p\left(x_{T} ; z\right)\right]+\tilde{g}_{T}[p(\bar{x} ; z)]
$$

for some continuous and positive functions $\tilde{f}_{1}, \tilde{f}_{2}, \ldots, \tilde{f}_{T}$, and $\tilde{g}_{T}$.

In the case where $x_{1}=x_{2}=\ldots=x_{T}$, denote $\xi=p\left(x_{t} ; z\right)=p(\bar{x} ; z)$, then the normalization axiom entails

$$
\sum_{t=1}^{T} \tilde{f}_{t}(\xi)+\tilde{g}_{T}(\xi)=\xi
$$

for all $\xi \in(0, p(0, z)]$. Differentiating equation (2.4) twice with respect to $\xi$ yields

$$
\sum_{t=1}^{T} \tilde{f}_{t}^{\prime \prime}(\xi)+\tilde{g}^{\prime \prime}(\xi)=0
$$

By choosing a $\mathbf{x}$ such that $x_{t}<z$ and $x_{s} \geq z$ for all $s \neq t$ with $\bar{x} \geq z$, equation (2.3) becomes

$$
P(\mathbf{x} ; z)=\tilde{f}_{t}\left[p\left(x_{t} ; z\right)\right] .
$$

The assumption $\frac{\partial^{2} P(\mathbf{x} ; z)}{\partial x_{t}^{2}} \geq 0$ implies

$$
\tilde{f}_{t}^{\prime \prime}\left(p^{\prime}\right)^{2}+\tilde{f}_{t}^{\prime} p^{\prime \prime} \geq 0 .
$$

Since the functional forms of $\tilde{f}_{1}, \tilde{f}_{2}, \ldots, \tilde{f}_{T}$, and $\tilde{g}_{T}$ are independent of the deprivation function $p$ used, by choosing a deprivation function $p$ such that $p^{\prime \prime}$ can be arbitrarily close to zero (say $p\left(x_{t} ; z\right)=z-x_{t}$ or better the index given in Zheng (1999, Equation (A3), p. 369)), we have

$$
\tilde{f}_{t}^{\prime \prime} \geq 0 \text { for all } t=1,2, \ldots, T \text {. }
$$

Next, choosing a $\mathbf{x}$ with $x_{1}>z$ but $\bar{x}<z$, we have

$$
P(\mathbf{x} ; z)=\tilde{f}_{1}[0]+\tilde{f}_{2}\left[p\left(x_{2} ; z\right)\right]+\ldots \tilde{f}_{T}\left[p\left(x_{T} ; z\right)\right]+\tilde{g}[p(\bar{x} ; z)] .
$$

Again, the requirement of $\frac{\partial^{2} P(\mathbf{x} ; z)}{\partial x_{1}^{2}} \geq 0$ implies

$$
\left[\tilde{g}^{\prime \prime}\left(p^{\prime}\right)^{2}+\tilde{g}^{\prime} p^{\prime \prime}\right] / n^{2} \geq 0,
$$


and once again it must be the case

$$
\tilde{g}^{\prime \prime} \geq 0
$$

This means all items in (2.5) cannot be negative. It follows that every one of them must be zero, or

$$
\tilde{f}_{t}(\xi)=f_{t}(t) \xi \text { for all } t=1,2, \ldots, T \text { and } \tilde{g}_{T}(\xi)=g(T) \xi
$$

for some continuous and positive functions $f_{t}(t), t=1,2, \ldots, T$, and $g(T)$. Substituting $\tilde{f}_{t}()$ and $\tilde{g}()$ back into $(2.3)$ completes the proof of the proposition.

Proposition 1 reveals that if a lifetime poverty index satisfies the three axioms proposed above, the marginal contributions from each period's poverty as well as from the poverty of average-lifetime-consumption are independent of the contributors. Since $\sum_{t=1}^{T} f_{t}(t)+g(T)=1$ by the normalization axiom and each coefficient is nonnegative and $g(T)>0$, we can further express (2.1) as

$$
P(\mathbf{x} ; z)=[1-g(T)]\left\{\sum_{t=1}^{T} \frac{f_{t}(t)}{1-g(T)} p\left(x_{t} ; z\right)\right\}+g(T) p(\bar{x} ; z) .
$$

Denote $\alpha(t, T)=\frac{f_{t}(t)}{1-g(T)}$ and $\beta(T)=1-g(T),(2.1 \mathrm{a})$ states that $P(\mathbf{x} ; z)$ is a weighted average between $\sum_{t=1}^{T} \alpha(t, T) p\left(x_{t} ; z\right)$ and $p(\bar{x} ; z)$ with the weights being $\beta(T)$ and $1-\beta(T)$, respectively. Note that $\sum_{t=1}^{T} \alpha(t, T) p\left(x_{t} ; z\right)$ is a weighted average of all snapshot-poverty levels of the $T$ periods with $\sum_{t=1}^{T} \alpha(t, T)=1$. This observation leads to the following corollary.

Corollary 2.1. A lifetime poverty measure $P(\mathbf{x} ; z)$ satisfies the strong monotonicity axiom, the independence axiom and the normalization axiom if and only if it can be written as a weighted average between the weighted average of all snapshot poverty levels and the poverty of average lifetime consumption

$$
P(\mathbf{x} ; z)=\beta(T)\left\{\sum_{t=1}^{T} \alpha(t, T) p\left(x_{t} ; z\right)\right\}+[1-\beta(T)] p(\bar{x} ; z) .
$$

for some $\alpha(t, T)$ and $\beta(T)$ such that $0<\alpha(t, T)<1,0<\beta(T)<1$ and $\sum_{t=1}^{T} \alpha(t, T)=$ 1 .

Examples of lifetime poverty indices include combinations of weight functions and snapshot poverty indices such as $\alpha(t, T)=\left(1-\frac{t}{T+1}\right)^{\gamma}$ or $\rho^{t}$ with $0<\rho<1$ and $p\left(x_{t} ; z\right)=\left(1-x_{t} / z\right)^{\varepsilon}, 1-\left(x_{t} / z\right)^{\varepsilon}$ and $\ln \left(z-x_{t}+1\right)$ for $x_{t}<z$.

The coefficient $\beta(T)$ plays the role of balancing between the average "snapshot poverty" and the average-lifetime-consumption or retrospective poverty: a larger value of $\beta(T)$ means that the individual is concerned more about the poverty incidences he has experienced and less about when the life as a whole is evaluated. In 
the limiting case of $\beta(T)=1$, the individual gives no consideration to the average or smoothed lifetime consumption; the individual's lifetime poverty in this case is determined exclusively by the poverty deprivations he has had in his life no matter how affluent he may be when life as a whole is judged. On the other hand, $\beta(T)=0$ means that the individual cares about only the lifetime aggregate or average consumption and the poverty deprivation in any period matters not at all in the evaluation of lifetime poverty. In this sense, we may label $\beta(T)$ as a "memory parameter" $\beta(T)=1$ is the polar case of "perfect memory" and $\beta(T)=0$ is the other polar case of "no memory," respectively. ${ }^{5}$ To compute the individual's lifetime poverty index using $(2.1 b)$ - which will be used in the rest of the paper, the memory parameter must be specified. If all possible values of $\beta(T)$ are considered, then we have

Corollary 2.2. For two lifetime consumption profiles $\mathbf{x}$ and $\mathbf{y}, P(\mathbf{x} ; z) \geq P(\mathbf{y} ; z)$ for any poverty measure of the form $(2.1 b)$ and for all $\beta(T) \in(0,1)$ if and only if

$$
\sum_{t=1}^{T} \alpha(t, T) p\left(x_{t} ; z\right) \geq \sum_{t=1}^{T} \alpha(t, T) p\left(y_{t} ; z\right) \text { and } p(\bar{x} ; z) \geq p(\bar{y} ; z) .
$$

This result can be regarded as our first dominance condition. The implication of the corollary is reasonable and intuitive. To characterize further the lifetime poverty index and establish additional dominance conditions, we need to take a closer look at the weighting function $\alpha(t, T)$. It is this task that we turn our attention to now.

For a given lifetime consumption profile, suppose the individual can choose a permutation of the $T$ consumption levels so as to minimize the lifetime poverty $P(\mathbf{x} ; z)$, what would the individual choose? Clearly, any permutation will not affect the poverty of average-lifetime-consumption since the average consumption remains the same. It is the average "snapshot" poverty that can be affected and the values of the coefficients $\alpha(t, T)$ dictate the chosen permutation. If $\alpha(t, T) \mathrm{s}$ are equal, i.e., $\alpha(s, T)=\alpha(t, T)$ for all $s$ and $t$, then any permutation of the consumption profile does not matter; otherwise, higher consumptions should be allocated to the periods with greater $\alpha$-values.

In general, higher values of $\alpha(t, T)$ reflect the periods that are more critical for an individual's lifetime well-being. If the individual (or ethical observer) cannot sort out the order of importance of all the periods, then there is no agreeable ranking for the $\alpha$-values. When each $\alpha(t, T)$ can assume any nonnegative value, we have the following result on the poverty orderings by all possible lifetime poverty measures (2.1b). The proof of the proposition is straightforward and is thus not furnished.

Proposition 2.2. For two lifetime consumption profiles $\mathbf{x}$ and $\mathbf{y}, P(\mathbf{x} ; z)>P(\mathbf{y} ; z)$

\footnotetext{
${ }^{5}$ By "perfect memory" we mean that the individual (or ethical observer) takes into account - and only takes into account - all the details involved with the pattern of lifetime consumptions while by "no memory" only the average lifetime consumption is used as a sufficient statistic to evaluate lifetime poverty.
} 
for all $\beta(T) \in[0,1]$ and all possible values of $\alpha(t, T) \mathrm{s}$ if and only if

$$
\tilde{x}_{t} \leq \tilde{y}_{t} \text { and } \min \{\bar{x}, z\} \leq \min \{\bar{y}, z\}
$$

for $t=1,2, \ldots, T$ and the strict inequality holds at least once. Note that $\tilde{x}_{t}=$ $\min \left\{x_{t}, z\right\}$.

Condition (2.8) states that for profile $\mathbf{x}$ to have more lifetime poverty than $\mathbf{y}$ unambiguously (i.e., for all possible weights), it must be the case that the consumption in each period of $\mathbf{x}$, if it is below the poverty line, is no greater than that in $\mathbf{y}$; moreover, it has also to be the case that the average lifetime consumption, if it is below the poverty line, is no greater in $\mathbf{x}$ than that in $\mathbf{y}$. Note that the first set of conditions does not necessarily imply the last condition because consumptions are censored at the poverty line.

Examples: For distributions of consumption $\mathbf{x}=(1,2,6)$ and $\mathbf{y}=(2,2,3)$ with $z=3$ note that the first set of conditions $\left(\tilde{x}_{t} \leq \tilde{y}_{t}\right)$ of Proposition 2.2 are satisfied, but not the second. Thus, for $\beta(T)$ small enough (i.e. $1-\beta(T)$ large enough), it is possible that $P(\mathbf{x} ; z) \leq P(\mathbf{y} ; z)$. To illustrate the importance of the condition that the poverty comparison is satisfied for all possible values of $\alpha(t, T)$, consider the following example with $z=5$ and $T=3$, with $\mathbf{x}=(4,3,1)$ and $\mathbf{y}=(1,4,4)$. Note that for equal weights $\alpha(t, T)$ we have $P(\mathbf{x} ; z)>P(\mathbf{y} ; z)$ since $p\left(x_{1} ; z\right)=p\left(y_{2} ; z\right), p\left(x_{2} ; z\right)>p\left(y_{3} ; z\right)$ and $p\left(x_{3} ; z\right)=p\left(y_{1} ; z\right)$. However, the greatest degree of poverty in the $\mathbf{x}$ distribution occurs earlier in life than for the $\mathbf{y}$ distribution. Applying the weights, for example, of $\rho^{t}$ for $\rho=0.8$ and using the poverty index $p(x ; z)=\max \{z-x, 0)$ we obtain $P(x ; z)<P(y ; z)$. Thus, we cannot order $\mathbf{x}$ and $\mathbf{y}$ unambiguously; i.e., for all values of weights $\alpha(t, T)$.

Numerical illustrations using PSID data: For all of our illustrations using the PSID (Panel Study of Income Dynamics) we follow families with heads who were included throughout all of the years 1968 to 2002. Although this doesn't represent full lifetimes for these individuals, this is one of the longest panel data sets available. Through the use of household equivalence scales and a normalization to 1983 dollars and the 1983 poverty line, we are able to standardize the experiences of poverty spells over this period. As a result of our selection criteria - i.e., the inclusion of only those families whose heads have lived over the entire period and continued to respond to the survey - we don't claim our sample to be representative. However, it is the relative numbers of pairwise comparisons that we can make as a result of our different combinations of axioms that is our interest, and this is satisfactorily illustrated with our selected sample. ${ }^{6}$ Our sample contains 670 individuals. Some individuals (approximately $2 / 3^{r d s}$ ) never experienced poverty and so lifetime poverty comparisons between such individuals are immaterial while any comparison between such a person who never has experienced poverty to a person who has experienced

\footnotetext{
${ }^{6}$ See the appendix for a more detailed description of the PSID data set and how we construct our comparisons.
} 
at least one poverty spell in his/her lifetime (the remaining $1 / 3^{r d}-218$ individuals) generates an obvious and unambiguous comparison for any of our sets of axioms regarding lifetime poverty. Therefore, we focus only on those 'interesting' pairwise comparisons involving individuals who have experienced at least one spell of poverty in their lifetimes $\left(C_{218}^{2}=23,653\right.$ pairs or combinations of 2$)$. Since our requirement in Proposition 2.2 is that some individual $A$ is poorer than another individual, $B$, iff $A$ has experienced at least as much poverty as $B$ in every period of life as well as for average lifetime income, it is perhaps not surprising that we can make a ranking in only $9 \%$ of such cases (2,118 of 23,653 pairwise comparisons). All of our following axioms and propositions involve some additional restriction(s) to the $\alpha(t, T) \mathrm{s}$ (weights over time periods) and so generate a larger fraction of rankings within the set of possible (interesting) pairwise comparisons.

Thus, our assumptions in proposition 2.2 represent the weakest set of restrictions on the set of admissible weight functions $\alpha(t, T) \mathrm{s}$ that we consider in this paper and the power of the ordering principle turns out to be quite weak when implementing pairwise comparisons based on any generally acceptable snapshot poverty index $p\left(x_{t} ; z\right)$. But this is not surprising. For example, with a poverty line of $z=5$ we cannot say which of the vector of incomes $\mathbf{x}=(1,6,10)$ and $\mathbf{y}=(6,4.9,10)$ reflects more lifetime poverty even though in the latter vector a person is only "slightly" poor in the second period of life while the first person sustains much more severe poverty even earlier in life. The reason for no unambiguous ranking is allowing for any pattern of $\alpha(t, T)$ s includes, for example, $\alpha(1, T)=0.01$ and $\alpha(2, T)=0.9($ and $\alpha(3, T)=0.09)$, which if applied to the poverty gap deprivation function $p\left(x_{t} ; z\right)=z-\widetilde{x}_{t}$, would judge $\mathbf{y}$ to be more poor than $\mathbf{x}$.

By contrast we make our strongest restriction on the pattern of admissible weight functions in the following proposition (2.3), namely that the individual concludes that all periods are equally important to him; i.e., $\alpha(s, T)=\alpha(t, T)$ for all $s$ and $t$. The following proposition can be easily verified using standard results from the literature of majorization.

Proposition 2.3. For two lifetime consumption profiles $\mathbf{x}$ and $\mathbf{y}$, if the $\alpha(t, T) \mathrm{s}$ are the same, then $P(\mathbf{x} ; z) \geq P(\mathbf{y} ; z)$ for all $\beta(T) \in[0,1]$ if and only if

(1) $\sum_{t=1}^{T} p\left(x_{t} ; z\right) \geq \sum_{t=1}^{T} p\left(y_{t} ; z\right)$ and $\min \{\bar{x}, z\} \leq \min \{\bar{y}, z\}$ for a given deprivation function $p$; or

(2) also for all deprivation functions $p$ such that $p^{\prime}<0$, vector $\left(\tilde{x}_{1}, \ldots, \tilde{x}_{T}\right)$ is rank dominated by vector $\left(\tilde{y}_{1}, \ldots, \tilde{y}_{T}\right)$ and $\min \{\bar{x}, z\} \leq \min \{\bar{y}, z\}$; or

(3) also for all deprivation functions $p$ such that $p^{\prime}<0$ and $p^{\prime \prime}>0$, vector $\left(\tilde{x}_{1}, \ldots, \tilde{x}_{T}\right)$ is generalized Lorenz dominated by vector $\left(\tilde{y}_{1}, \ldots, \tilde{y}_{T}\right)$ and $\min \{\bar{x}, z\} \leq$ $\min \{\bar{y}, z\}$.

The implications of requiring weights $\alpha(t, T)$ to be equal for all $t$ leads to a large fraction of cases to be ranked in our PSID data set. For all deprivation functions that satisfy $p^{\prime}<0$, we find $63 \%$ of pairwise comparisons ranked. If one adds the requirement that $p^{\prime \prime}>0$, which is commonly assumed in the literature on poverty 
indices, we find $73 \%$ are ranked.

One can think of the assumptions made in Proposition 2.2 and 2.3 as extreme cases of weakness and strength regarding permissible patterns of the weight functions $\alpha(t, T)$ for comparing the importance of the timing of poverty in terms of its impact on lifetime poverty. There is clearly a dramatic loss of power in making pairwise rankings by moving from the strongest to weakest set of restrictions. In what follows we consider intermediate positions which we argue reflect received concerns about relative timing of poverty in an individual's life. If the individual (or ethical observer) can successfully rank order the importance of his well-being in all periods with no two periods equally important, ${ }^{7}$ then condition (2.8) can be refined. As noted in the introduction, it seems that there is a consensus that early stages of life such as childhood matter more than later-life periods in shaping the individual's lifetime well-being/poverty. It can be argued that the more poverty that a person suffers in his childhood or less education he receives in his youth, the less is the person's future well-being conditional on any given level of consumption. There are several reasons for such an effect. For example, poorer health or poorer psychological well-being including the effect that arises from less education. Of course, there are also functional implications of early poverty on future consumption due to reduced capabilities. As noted earlier, this impact is already taken into account in our measurement of lifetime consumption through the inclusion of the entire lifetime consumption vector in $P(\mathbf{x} ; z)$. Naturally, consumption and poverty later in life cannot impact well-being earlier in life. Translated in terms of the weighting function $\alpha(t, T)$, this notion can be formally stated as an axiom.

The early-poverty axiom: the weighting function $\alpha(t, T)$ is nonincreasing in time $t$.

Note that what the early-poverty axiom states is different from the discounting concern that is usually imposed on aggregation over time, although the discounting weight function $\alpha(t, T)=\rho^{t}$ with $0<\rho<1$ does happen to satisfy the axiom. Here we do not discount over time per se; our concern is purely about the size of impact of each period's poverty deprivation on the aggregate lifetime poverty. In fact, as we will see later, the usual discount-weighting scheme is ruled out when a further axiom is introduced. As for the other type of weighting function mentioned earlier, i.e., $\alpha(t, T)=\left(1-\frac{t}{T+1}\right)^{\gamma}, \gamma>0$ is required for it to satisfy the early-poverty axiom. ${ }^{8}$ With this additional axiom, we have

Proposition 2.4. For two lifetime consumption profiles $\mathbf{x}$ and $\mathbf{y}, P(\mathbf{x} ; z) \geq P(\mathbf{y} ; z)$ for all $\beta(T) \in[0,1]$ and all possible values of $\alpha(t, T)$ s satisfying the early-poverty

\footnotetext{
${ }^{7}$ If any two or more periods are equally important, i.e., their coefficients are the same, then these periods can be grouped together to form a single period and the results derived in Proposition 2.3 and onward apply to this "grouped" consumption profile.

${ }^{8}$ Any given set of weights $\alpha(t, T)$ can be normalized by simply dividing by the sum in order to ensure $\sum_{t=1}^{T} \alpha(t, T)=1$. Also, a positive constant could be added to $\alpha(t, T)$ for the above example in a preliminary step in order to ensure $\alpha(t, T)>0$ for $t=T$
} 
axiom if and only if

(1) $\sum_{t=1}^{l} p\left(x_{t} ; z\right) \geq \sum_{t=1}^{l} p\left(y_{t} ; z\right)$ for $l=1,2, \ldots, T$ and $\min \{\bar{x}, z\} \leq \min \{\bar{y}, z\}$ for a given deprivation function $p$; or

(2) also for all deprivation functions $p$ such that $p^{\prime}<0$, vector $\left(\tilde{x}_{1}, \ldots, \tilde{x}_{l}\right)$ is rank dominated by vector $\left(\tilde{y}_{1}, \ldots, \tilde{y}_{l}\right)$ for $l=1,2, \ldots, T$ and $\min \{\bar{x}, z\} \leq \min \{\bar{y}, z\}$; or

(3) also for all deprivation functions $p$ such that $p^{\prime}<0$ and $p^{\prime \prime}>0$, vector $\left(\tilde{x}_{1}, \ldots, \tilde{x}_{l}\right)$ is generalized Lorenz dominated by vector $\left(\tilde{y}_{1}, \ldots, \tilde{y}_{l}\right)$ for $l=1,2, \ldots, T$ and $\min \{\bar{x}, z\} \leq \min \{\bar{y}, z\}$.

Proof. The early-poverty axiom is equivalent to requiring $\alpha(1, T) \geq \alpha(2, T) \ldots \geq$ $\alpha(T, T)$. With this condition, the proof of part (1) follows directly from Abel's partial summation formula (see Rudin (1976, p. 70)). Parts (2) and (3) result from applying the standard rank order condition (Saposnik, 1986) and majorization (Marshall and Olkin, 1979).

A necessary condition for parts (2) and (3) in the above proposition is

$$
\sum_{t=1}^{l} \tilde{x}_{t} \leq \sum_{t=1}^{l} \tilde{y}_{t} \text { for } l=1,2, \ldots, T
$$

which is the concentration curve dominance between (censored) lifetime consumption profiles of $\mathbf{x}$ and $\mathbf{y}$. Concentration curve dominance is constructed similarly to the generalized Lorenz curve dominance with the exception that the values of $\left\{x_{t}\right\}$ and $\left\{y_{t}\right\}$ are not sorted before the construction. The concentration curve dominance condition can be handily used to screen out consumption profiles in (lifetime) poverty orderings.

Example and empirical illustration: Adopting the early poverty axiom is sufficient to allow for an ordering between $\mathbf{x}=(1,6,10)$ and $\mathbf{y}=(6,4.9,10)$, with poverty line $z=5$, unlike the case when the pattern of the $\alpha(t, T)$ s was not restricted in any way (i.e., by Proposition 2.2); that is, the early poverty axiom implies the trade-off that the poverty in the first period of life for profile $\mathbf{x}$ is 'more important' than the poverty experienced in the second period of life in profile, $\mathbf{y}$. In fact, even $\mathbf{x}=(1,6,10)$ and $\mathbf{y}=(6,1,10)$ would be ordered accordingly by the early poverty axiom. Our comparisons using the PSID data set suggests the early poverty axiom may lead to a substantial increase in the ability to make pairwise rankings in real world applications. Upon doing these comparisons we find that $38 \%$ of the 'interesting' cases are ranked for result (2) - when $p^{\prime}<0$ only is assumed - while $42 \%$ are decided for result (3) when $p^{\prime \prime}<0$ is also assumed.

Finally, we introduce the axiom that characterizes the chronic aspect of lifetime poverty. Suppose an individual has to endure two poverty spells within certain periods of time. Would his lifetime poverty be greater if he had to live in poverty consecutively for two periods or alternatively with a period of affluence in between? Chronic poverty is generally defined as living in poverty continuously for an extended period of time. The issue of chronic poverty has been at the heart of recent poverty research and 
anti-poverty policy debate (see, for example, Hulme and McKay, 2005; the Chronic Poverty Research Center Report, 2005). It is argued that transitory poverty is not as much a cause for concern as chronic poverty. Chronic poverty should be the target for poverty reduction effort because it gives rise to a series of social-economic problems and poses the greater threat to economic growth in developing countries. Following this notion, we argue that living in poverty for consecutive periods leads to higher lifetime poverty than living in poverty and prosperity alternatively even though the total number of poverty spells and depth of poverty remain the same. This consideration is formally presented in the axiom below.

The chronic-poverty axiom: for two given consumptions $a<z$ and $b<z$, the closer the two spells together, the greater is the resulting lifetime poverty, i.e.,

$$
\alpha(s, T) p(a ; z)+\alpha(u, T) p(b ; z) \geq \alpha(r, T) p(a ; z)+\alpha(v, T) p(b ; z)
$$

for all $1 \leq r<s \leq u<v \leq T$ such that $s-r=v-u$.

Notice that the chronic-poverty axiom specifies that two spells of poverty occurring in periods $(s, u)$ have greater impact than if the same spells had occurred in periods spread out symmetrically by $k>0$ periods in both directions; i.e., in periods $(r, v)=$ $(s-k, u+k)$. To understand the reason for adopting this symmetry requirement, consider the implication of not doing so and letting $s-r \neq v-u$. Specifically, consider the case of $r=1, s=u=T-1$, and $v=T$ for $T>3$. Set $a=b$ and note that assuming the chronic-poverty axiom without the normalization of symmetry, $s-r=v-u$, would lead to

$$
\alpha(T-1, T) p(a ; z)+\alpha(T-1, T) p(a ; z) \geq \alpha(1, T) p(a ; z)+\alpha(T, T) p(a ; z)
$$

Dividing by $p(a ; z)$ and re-arranging gives the inequality

$$
\alpha(1, T)-\alpha(T, T) \leq 2[\alpha(T-1, T)-\alpha(T, T)]
$$

Therefore, without the requirement $s-r=v-u$ the early poverty axiom would be compromised to the extent that the range of the (nonincreasing) weights over the entire time period $T=1$ to $T=T$ would be restricted to only twice the difference between the weights assigned to the last two time periods. Thus, if the weights for the last two time periods were equal then the "chronic-poverty axiom" without requiring $s-r=v-u$ would imply that the early poverty axiom would be made impotent (i.e., the weights $\alpha(t, T)$ would have to be equal for all $t$ ).

The chronic-poverty axiom (with the requirement of $s-r=v-u$ ) implies that the weighting function $\alpha(t, T)$ is concave in $t .{ }^{9}$ To see this take the case of $a=b$ in (2.9) to obtain

$$
\alpha(s, T) p(a ; z)+\alpha(u, T) p(a ; z) \geq \alpha(r, T) p(a ; z)+\alpha(v, T) p(a ; z)
$$

\footnotetext{
${ }^{9}$ Recall that the early poverty axiom implies that the weights $\alpha(t, T)$ are non-increasing in $t$.
} 
Dividing by $p(a ; z)$ and rearranging gives

$$
\alpha(s-k, T)-\alpha(s, T) \leq \alpha(u, T)-\alpha(u+k, T), u \geq s
$$

which is satisfied if and only if $\alpha(t, T)$ is concave in $t$.

To fully adopt the chronic-poverty axiom for poverty measurement, we need to extend the weighting function to period $T+1: \alpha(T+1, T)=0$. This is a sort of assumption of "poverty irrelevance of life after death." By letting $a=b$ and iteratively $s=u=2, \ldots, T$ and choosing $k=1$ (2.9a), we have

$$
0<\alpha(1, T)-\alpha(2, T) \leq \ldots \leq \alpha(T-1, T)-\alpha(T, T) \leq \alpha(T, T)
$$

Examples of satisfactory poverty indices: $\alpha(t, T)=\left(1-\frac{t}{T+1}\right)^{\gamma}$ with $0<\gamma<1$ and $\alpha(t, T)=1-\left(\frac{t}{T+1}\right)^{\gamma}$ with $\gamma>1$. The discount-rate coefficient $\alpha(t, T)=\rho^{t}$ with $0<\rho<1$ does not satisfy the chronic poverty axiom since it is convex in $t$.

Rewrite (2.1b) as

$$
\begin{aligned}
P(\mathbf{x} ; z)= & \beta(T)\left\{\left[\sum_{t=1}^{T-1}[\alpha(t, T)-\alpha(t+1, T)] \sum_{s=1}^{t} p\left(x_{s} ; z\right)\right]+\alpha(T, T) \sum_{s=1}^{T} p\left(x_{s} ; z\right)\right\} \\
& +[1-\beta(T)] p(\bar{x} ; z) .
\end{aligned}
$$

and use Abel's partial summation formula one more time (but in reserve order), we have:

Proposition 2.5. For two lifetime consumption profiles $\mathbf{x}$ and $\mathbf{y}, P(\mathbf{x} ; z) \geq P(\mathbf{y} ; z)$ for all $\beta(T) \in[0,1]$ and all possible values of $\alpha(t, T)$ s satisfying the early-poverty axiom and the chronic-poverty axiom if and only if

(1) $l \sum_{t=1}^{T-l+1} p\left(x_{l}, z\right)+\sum_{m=1}^{l-1}(l-m) p\left(x_{T-l+m+1}, z\right) \geq l \sum_{t=1}^{T-l+1} p\left(y_{l}, z\right)+\sum_{m=1}^{l-1}(l-$ m) $p\left(y_{T-l+m+1}, z\right)$ for $l=1,2, \ldots, T$ and $\min \{\bar{x}, z\} \leq \min \{\bar{y}, z\}$ for a given deprivation function $p$; or

(2) also for all deprivation functions $p$ such that $p^{\prime}<0$, vector

$$
\tilde{\mathbf{x}}_{l}=\left(\tilde{x}_{1}, . ., \tilde{x}_{1}, \ldots, \tilde{x}_{T-l+1}, . ., \tilde{x}_{T-l+1}, \tilde{x}_{T-l}, . ., \tilde{x}_{T-l}, \tilde{x}_{T-l}, . ., \tilde{x}_{T-l}, \ldots, \tilde{x}_{T}\right)
$$

is rank dominated by a similarly defined vector $\tilde{\mathbf{y}}_{l}$ for $l=1,2, \ldots, T$ and $\min \{\bar{x}, z\} \leq$ $\min \{\bar{y}, z\} ;{ }^{10}$ or

(3) also for all deprivation functions $p$ such that $p^{\prime}<0$ and $p^{\prime \prime}>0$, vector $\tilde{\mathbf{x}}_{l}$ is generalized Lorenz dominated by vector $\tilde{\mathbf{y}}_{l}$ for $l=1,2, \ldots, T$ and $\min \{\bar{x}, z\} \leq$ $\min \{\bar{y}, z\}$.

Note that a dominance between $\tilde{\mathbf{x}}_{l}$ and $\tilde{\mathbf{y}}_{l}$ does not imply nor is implied by the dominance between $\left(\tilde{x}_{1}, . ., \tilde{x}_{T}\right)$ and $\left(\tilde{y}_{1}, . ., \tilde{y}_{T}\right)$ because none of the vectors is ordered (if they are increasingly ordered then the two types of dominance would be equivalent).

\footnotetext{
${ }^{10}$ See appendix for elaboration on how this set of vectors is constructed.
} 
To see this, consider $\mathbf{x}=(3,1)$ and $\mathbf{y}=(2,4)$ with $z=5$. Clearly $\mathbf{x}$ is rank ordered by $\mathbf{y}$ but $\tilde{\mathbf{x}}_{2}=(3,3,1)$ is not rank ordered by $\tilde{\mathbf{y}}_{2}=(2,2,4)$.

Empirical illustration: Adding the chronic poverty axiom improves the power of the ranking principle in much the same way as does adding risk aversion $\left(u^{\prime \prime}<0\right)$ to the monotonicity axiom $\left(u^{\prime}>0\right)$ in expected utility theory. So, in Proposition 2.5 the set of permissible weights $\alpha(t, T) \mathrm{s}$ must be nonincreasing (early poverty axiom) and concave (chronic poverty axiom) in $t$. Our comparisons using the PSID data set suggests the combination of the early poverty and chronic poverty axioms may lead to a substantial increase the ability to make pairwise rankings in real world applications relative to no restrictions whatsoever. Upon doing these comparisons we find that $46 \%$ of the 'interesting' cases are ranked for result (2) - when $p^{\prime}<0$ only is assumed - and $63 \%$ are decided for result $(3)$ - when $p^{\prime \prime}<0$ is also assumed. These compare to $38 \%$ and $42 \%$, respectively, when only the early poverty axiom is adopted. ${ }^{11}$

\section{The Measurement of a Society's Lifetime Poverty}

Consider a society consisting of $N$ individuals who live through the same $T$ periods. These individuals are identical except that they have different lifetime consumption profiles. For each individual $i$, his consumption profile is $\mathbf{x}^{i}=\left(x_{1}^{i}, \ldots, x_{t}^{i}, \ldots x_{T}^{i}\right)^{\prime}$ and for each period $t$, the society's consumption distribution is $\mathbf{x}_{t}=\left(x_{t}^{1}, \ldots, x_{t}^{i}, \ldots x_{t}^{N}\right)$. The society's lifetime consumption data is represented by a $N \times T$ matrix $X=$ $\left(\mathbf{x}^{1}, \ldots, \mathbf{x}^{N}\right)=\left(\mathbf{x}_{1}, \ldots, \mathbf{x}_{T}\right)^{\prime}$. In each period, each individual's poverty status is determined by comparing his consumption level with the poverty line $0<z<\infty$ which is exogenously given and remains constant throughout the $T$ periods. An individual is poor in period $t$ if and only if his consumption $x_{t}$ is strictly less than $z$. An individual's lifetime poverty is $P\left(\mathbf{x}^{i} ; z\right)$ and for each period the society's poverty is $P\left(\mathbf{x}_{t} ; z\right)$. The society's lifetime poverty level is determined via a function $P(X ; z)$ which maps each consumption matrix of the society $X$ into $[0, \infty)$. The average consumption level of individual $i$ over the $T$ periods is $\bar{x}^{i}$ and the average consumption level of the society at time $t$ is $\bar{x}_{t}$. As before, we assume that the lifetime poverty measure $P(X ; z)$ exhibits properties $\frac{\partial P(X ; z)}{\partial x_{t}^{i}} \leq 0$ and $\frac{\partial^{2} P(X ; z)}{\partial\left(x_{t}^{i}\right)^{2}} \geq 0$ for all $x_{t}^{i} \in[0, \infty)$.

Conceptually, there are two routes to aggregate individual deprivations into a society's lifetime poverty value. The first approach - the person-first approach - is to aggregate each individual's lifetime poverty first - as we have done in the previous section - and then to aggregate across all individuals. Following this approach, the society's lifetime poverty $P(X ; z)$ can be written as

$$
P(X ; z)=\tilde{h}\left[P\left(\mathbf{x}^{1} ; z\right), \ldots, P\left(\mathbf{x}^{N} ; z\right)\right]
$$

for some continuous function $\tilde{h}()$ which reflects society's preference about the distribution of lifetime poverty deprivations. The second approach - the society-first

\footnotetext{
${ }^{11} \mathrm{~A}$ fuller discussion of the procedures for making comparisons under the different Propositions and a table summarizing our empirical illustrations are provided in the appendix.
} 
approach - is to aggregate society's poverty deprivation in each period by aggregating across individuals and then aggregate these deprivations across all $T$ periods. In this approach, the aggregation of poverty in each period follows the standard approach outlined in the literature of snapshot-poverty measurement and the resulting index satisfies all the basic axioms. The second stage of the aggregation follows the steps that we have characterized in the previous section of the paper. In general, we can write the poverty measure derived with the second approach as

$$
P(X ; z)=k\left[P\left(\mathbf{x}_{1} ; z\right), \ldots, P\left(\mathbf{x}_{T} ; z\right)\right]
$$

for some continuous function $k()$ which also reflects society's preference on aggregation over time. Which approach is better? It seems that both are sensible and each can be favorably argued on different grounds, but the two approaches obviously will not always depict the same picture for society's lifetime poverty and its changes.

A natural question is when will both approaches be consistent in depicting the poverty picture of a society over the $T$ periods? Or, in other words, when will the two routes of aggregation lead to the same result? The following path-independence axiom ensures the consistency between the two approaches.

The path-independence axiom. For any consumption matrix $X$, both the personfirst approach and the society-first approach should yield the same level of lifetime poverty for the society.

In aggregating poverty across a population, the axiom of decomposability is commonly used. This axiom allows the overall poverty value to be a weighted average of the poverty levels of subpopulations with the weights being the proportions of population subgroups. Applying such a decomposability requirement to $P(X ; z)$, we have for the person-first approach,

$$
P(X ; z)=h\left[P\left(\mathbf{x}^{1} ; z\right)\right]+\ldots+h\left[P\left(\mathbf{x}^{N} ; z\right)\right]
$$

where $h()$ is a continuous function which reflects society's preference on the aggregation process. We require function $h$ to preserve the origin, i.e., $h(0)=0$ since $P(X ; z)=0$ if $x_{t}^{i} \geq z$ for all $i$ and $t$. This decomposability property is also applicable to the aggregation of society's poverty in each period. For the case of "perfect memory," i.e., $\beta(T)=1$, we should have

$$
P\left(\mathbf{x}_{t} ; z\right)=h\left[p\left(x_{t}^{1} ; z\right)\right]+\ldots+h\left[p\left(x_{t}^{N} ; z\right)\right] .
$$

With decomposability, we can characterize a class of path-independent measures of lifetime poverty.

Proposition 3.1. A lifetime poverty measure $P(X ; z)$ satisfies both the decomposability axiom and the path-independence axiom if and only if $P(X ; z)$ is a positive multiple of

$$
P(X ; z)=\beta(T)\left\{\sum_{t=1}^{T} \alpha(t, T) \sum_{i=1}^{N} p\left(x_{t}^{i} ; z\right)\right\}+[1-\beta(T)] \sum_{i=1}^{N} p\left(\bar{x}^{i} ; z\right) .
$$


Proof. For simplicity, consider $T=N=2$. The path-independence axiom requires that

$$
P(X ; z)=\tilde{h}\left[P\left(\mathbf{x}^{1} ; z\right), P\left(\mathbf{x}^{2} ; z\right)\right]=k\left[P\left(\mathbf{x}_{1} ; z\right), P\left(\mathbf{x}_{2} ; z\right)\right] .
$$

Let $\beta(T)=1$, then with (2.1b) and the decomposability axiom, (3.4) becomes

$$
\begin{aligned}
& h\left[\sum_{t=1}^{2} \alpha(t, T) p\left(x_{t}^{1} ; z\right)\right]+h\left[\sum_{t=1}^{2} \alpha(t, T) p\left(x_{t}^{2} ; z\right)\right] \\
= & k\left\{\sum_{i=1}^{2} h\left[p\left(x_{1}^{i} ; z\right)\right], \sum_{i=1}^{2} h\left[p\left(x_{2}^{i} ; z\right)\right]\right\} .
\end{aligned}
$$

Let $x_{2}^{1}=z$ and $x_{2}^{2}=z$ so that $p\left(x_{2}^{1} ; z\right)=p\left(x_{2}^{2} ; z\right)=0$, then (3.5) becomes

$$
h\left[\alpha(1, T) p\left(x_{1}^{1} ; z\right)\right]+h\left[\alpha(1, T) p\left(x_{1}^{2} ; z\right)\right]=k\left\{h\left[p\left(x_{1}^{1} ; z\right)\right]+h\left[p\left(x_{1}^{2} ; z\right)\right], 0\right\} .
$$

Denote $\omega_{1}=\alpha(1, T) p\left(x_{1}^{1} ; z\right), \omega_{2}=\alpha(1, T) p\left(x_{1}^{2} ; z\right)$ and $\tilde{k}\left(\omega_{1}+\omega_{2}\right)=k\left\{h\left[\omega_{1} / a(1, T)\right]+\right.$ $h\left[\omega_{2} / a(1, T], 0\right\}$, we have

$$
h\left(\omega_{1}\right)+h\left(\omega_{2}\right)=\tilde{k}\left(\omega_{1}+\omega_{2}\right)
$$

for all $\omega_{1}$ and $\omega_{2}$ in $[0, a(1, T) p(0 ; z)]$ which is non-empty since both $a(1, T)$ and $p(0 ; z)$ are strictly positive.

Equation (3.6) is a Pexider equation and its nontrivial solution (Aczél, 1966, p. 142, Theorem 1 and Corollary 1) is:

$$
h\left(\omega_{1}\right)=a \omega_{1}+b
$$

for some constants $a$ and $b$. But because $h^{\prime}>0$ and $h(0)=0, b$ must be 0 and $a>0$. Substituting $h\left(\omega_{1}\right)=a \omega_{1}$ into (3.1a) and using (2.1c), (3.1b) is derived.

When all possible "memory parameter" $\beta(T) \mathrm{s}$, the weighting coefficients $\alpha(t, T)$ and/or individual deprivation functions $p(x ; z)$ are considered, we have the following results on societal lifetime poverty orderings.

Proposition 3.2. For two lifetime consumption profiles $X$ and $Y$ and a poverty measure $P()$ given in $(3.1 \mathrm{~b}), P(X ; z) \geq P(Y ; z)$ for all $\beta(T) \in[0,1]$ and all possible values of $\alpha(t, T)$ s satisfying the early-poverty axiom and the chronic-poverty axiom if and only if

(1)

$$
\begin{aligned}
& l \sum_{t=1}^{T-l+1} \sum_{i=1}^{N} p\left(x_{l}^{i} ; z\right)+\sum_{m=1}^{l-1}(l-m) \sum_{i=1}^{N} p\left(x_{T-l+m+1}^{i} ; z\right) \\
\geq & l \sum_{t=1}^{T-l+1} \sum_{i=1}^{N} p\left(y_{l}^{i} ; z\right)+\sum_{m=1}^{l-1}(l-m) \sum_{i=1}^{N} p\left(y_{T-l+m+1}^{i} ; z\right)
\end{aligned}
$$


for $l=1,2, \ldots, T$ and $\sum_{i=1}^{N} p\left(\bar{x}^{i} ; z\right) \leq \sum_{i=1}^{N} p\left(\bar{y}^{i} ; z\right)$ for a given deprivation function $p$; or

(2) also for all deprivation functions $p$ such that $p^{\prime}<0$, vector

$$
\tilde{X}_{l}=\left(\tilde{\mathbf{x}}_{1}, . ., \tilde{\mathbf{x}}_{1}, \ldots, \tilde{\mathbf{x}}_{T-l+1}, . ., \tilde{\mathbf{x}}_{T-l+1}, \tilde{\mathbf{x}}_{T-l}, . ., \tilde{\mathbf{x}}_{T-l}, \tilde{\mathbf{x}}_{T-l}, . ., \tilde{\mathbf{x}}_{T-l}, \ldots, \tilde{\mathbf{x}}_{T}\right)
$$

is rank dominated by a similarly defined vector $\tilde{Y}_{l}$ for $l=1,2, \ldots, T$ and $\left(\min \left\{\bar{x}^{1}, z\right\}, \ldots\right.$, $\left.\min \left\{\bar{x}^{N}, z\right\}\right)$ is rank ordered by $\left(\min \left\{\bar{y}^{1}, z\right\}, \ldots, \min \left\{\bar{y}^{N}, z\right\}\right)$; or

(3) also for all deprivation functions $p$ such that $p^{\prime}<0$ and $p^{\prime \prime}>0$, vector $\tilde{X}_{l}$ is generalized Lorenz dominated by vector $\tilde{Y}_{l}$ for $l=1,2, \ldots, T$ and $\left(\min \left\{\bar{x}^{1}, z\right\}, \ldots, \min \left\{\bar{x}^{N}, z\right\}\right)$ is generalized Lorenz dominated by $\left(\min \left\{\bar{y}^{1}, z\right\}, \ldots, \min \left\{\bar{y}^{N}, z\right\}\right)$.

Proof. All results can be derived similarly to those in the previous section and thus are omitted.

In measuring a society's lifetime poverty, as in any other social welfare measurement over time, income/consumption mobility should be an important factor. It is useful to point out that such a consideration of consumption mobility is contained in the second condition in each case of the proposition. The second condition is the comparison of dominance between two censored mean consumption profiles, i.e., between $\left(\min \left\{\bar{x}^{1}, z\right\}, \ldots, \min \left\{\bar{x}^{N}, z\right\}\right)$ and $\left(\min \left\{\bar{y}^{1}, z\right\}, \ldots, \min \left\{\bar{y}^{N}, z\right\}\right)$. To see this and to connect with the literature of mobility measurement, it is necessary to introduce the notation of joint distribution among consumptions in different periods. Denote $\left(x_{1}, \ldots, x_{t}, \ldots, x_{T}\right)$ as the consumption profile of a representative individual, $F\left(x_{1}, \ldots, x_{t}, \ldots x_{T}\right)$ as the joint cdf of one society's consumptions over the $T$ periods and $G\left(x_{1}, \ldots, x_{t}, \ldots x_{T}\right)$ as the joint cdf of another society's consumptions. Suppose $\left(\min \left\{\bar{x}^{1}, z\right\}, \ldots, \min \left\{\bar{x}^{N}, z\right\}\right)$ is rank dominated by $\left(\min \left\{\bar{y}^{1}, z\right\}, \ldots, \min \left\{\bar{y}^{N}, z\right\}\right)$, then it is equivalent that $\min \{\bar{y}, z\}$ first-degree stochastic dominates $\min \{\bar{x}, z\}$. It is easy to see that this last dominance is further equivalent to

$$
F\left(x_{1}, \ldots, x_{t}, \ldots x_{T}\right) \geq G\left(x_{1}, \ldots, x_{t}, \ldots x_{T}\right) \text { for all }\left(x_{1}, \ldots, x_{t}, \ldots x_{T}\right) \text { such that } \bar{x}<z .
$$

For readers who are familiar with the literature of income mobility (e.g., Atkinson and Bourguignon, 1982, Dardanoni, 1993), condition (3.7) is a limited first-degree mobility dominance condition applied to the region of $\left\{\left(x_{1}, \ldots, x_{t}, \ldots x_{T}\right) \mid \bar{x}<z\right\}$. This mobility factor requires that the aggregation of individual lifetime poverty satisfy a limited version of the familiar "equality-preferring axiom" - the society prefers the individual poverty deprivations to be equally distributed. This is because, all else the same, a more equal distribution of $\left(\min \left\{\bar{x}^{1}, z\right\}, \ldots, \min \left\{\bar{x}^{N}, z\right\}\right)$ leads to a lower societal aggregate lifetime poverty.

The first condition (i.e., dominance between $\tilde{X}_{l}$ and $\tilde{Y}_{l}$ for $l=1,2, \ldots, T$ ) does not contain any element of mobility. This is because in (3.1b), individual poverty deprivations are aggregated across society in each time period. As a result, there is no way to trace any individual's consumption over time. The dominance relations between $\tilde{X}_{l}$ and $\tilde{Y}_{l}$ are related to the dominances of marginal cdfs such as 
$F\left(x_{1}, \infty, \ldots, \infty\right) \geq G\left(x_{1}, \infty, \ldots, \infty\right)$ for $x_{1}<z$. Clearly, this type of dominance among the marginal cdfs does not concern income mobility at all. It is also clear that the dominances of marginal cdfs and (3.7) do not imply each other for $z<\infty$. But as is well known, if $z=\infty$, then condition (3.7) will necessarily imply all the dominances among marginal cdfs such as $F\left(x_{1}, \infty, \ldots, \infty\right)$ and $F\left(x_{1}, x_{2}, \infty, \ldots, \infty\right)$.

But if we follow the person-first approach of aggregation and let the aggregating operator $h()$ in (3.1a) satisfy $h^{\prime}>0$ and $h^{\prime \prime}>0$, i.e., requiring the equity axiom at the outset, consumption mobility will play a role in lifetime poverty comparison even if only snapshot poverty levels are aggregated. To see this, consider $T=2$ and let $\beta(T)=1$, then (3.1a) becomes

$$
P(X ; z)=\int_{0}^{\infty} \int_{0}^{\infty} h\left[\alpha(1,2) p\left(x_{1} ; z\right)+\alpha(2,2) p\left(x_{2} ; z\right)\right] d F\left(x_{1}, x_{2}\right) .
$$

Integrating $P(X ; z)$ by parts twice (similar to what is done in Atkinson and Bourguignon (1982)) and using the condition $p\left(x_{t} ; z\right)=0$ for $x_{t} \geq z$, we have

$$
\begin{aligned}
P(X ; z)= & -\int_{0}^{z}\left\{h^{\prime} \alpha(1,2) p^{\prime}\left(x_{1} ; z\right)\right\} F\left(x_{1}, \infty\right) d x_{1}-\int_{0}^{z} h^{\prime}\left\{\alpha(2,2) p^{\prime}\left(x_{2} ; z\right)\right\} F\left(\infty, x_{2}\right) d x_{2} \\
& +\int_{0}^{2 z} \int_{0}^{2 z-x_{1}}\left\{h^{\prime \prime} \alpha(1,2) \alpha(2,2) p^{\prime}\left(x_{1} ; z\right) p^{\prime}\left(x_{2} ; z\right)\right\} F\left(x_{1}, x_{2}\right) d x_{2} d x_{1} .
\end{aligned}
$$

Since $h^{\prime}>0$ and $h^{\prime \prime}>0$, then for all possible values of $\alpha(1,2)$ and $\alpha(2,2)$, the necessary and sufficient condition for lifetime poverty to be higher in distribution $F$ than in $G$ for all poverty measures (3.1b) with $p^{\prime}<0$ would be

$$
F\left(x_{1}, \infty\right) \geq G\left(x_{1}, \infty\right) \text { for all } x_{1}<z ; F\left(\infty, x_{2}\right) \geq G\left(\infty, x_{2}\right) \text { for all } x_{2}<z
$$

and $F\left(x_{1}, x_{2}\right) \geq G\left(x_{1}, x_{2}\right)$ for all $x_{1}$ and $x_{2}$ such that $x_{1}+x_{2}<2 z$.

If $\alpha(1,2) \geq \alpha(2,2)$, then for all possible such values of $\alpha(1,2)$ and $\alpha(2,2)$, the necessary and sufficient condition for lifetime poverty to be higher in distribution $F$ than in $G$ for all poverty measures (3.1b) with $p^{\prime}<0$ would be

$$
\begin{aligned}
F\left(x_{1}, \infty\right) & \geq G\left(x_{1}, \infty\right) \text { for all } x_{1}<z ; \\
F\left(x_{1}, \infty\right)+F\left(\infty, x_{1}\right) & \geq G\left(x_{1}, \infty\right)+G\left(\infty, x_{1}\right) \text { for all } x_{1}<z ; \\
\text { and } F\left(x_{1}, x_{2}\right) & \geq G\left(x_{1}, x_{2}\right) \text { for all } x_{1} \text { and } x_{2} \text { such that } x_{1}+x_{2}<2 z
\end{aligned}
$$

In both sets of conditions, consumption mobility is reflected in the requirement $F\left(x_{1}, x_{2}\right) \geq G\left(x_{1}, x_{2}\right)$ over $x_{1}+x_{2}<2 z$. Note that these dominance conditions can be generalized to more than two periods and for all poverty deprivation functions satisfying $p^{\prime}<0$ and $p^{\prime \prime}>0$, but additional conditions of higher orders (all the way up to the $2 T$ th order) must be specified on the $h$ function. Although the PSID data set proves useful for illustrating the relative power of alternative sets of axioms to generate pairwise comparisons, it does not provide useful alternative 'societies' for doing 
so at this level of aggregation. The framework in this section, however, should prove useful in applications of measuring societal lifetime poverty when comparing a pair of societies or hypothetical outcomes for societies of alternative poverty alleviation strategies

\section{Some Remarks and Conclusion}

Our formulation of lifetime poverty measurement provides an axiomatic framework applied to poverty experiences over multiple periods of an individual's life. Besides recognizing that an individual's lifetime poverty should be reflected both by poverty experiences of each period (i.e., "snapshot" poverty) and the poverty level of "permanent" or retrospective lifetime consumption, we also introduce axioms to reflect the sensitivity of how any poverty spells are distributed over a person's lifetime. In particular, we investigate the implications of adopting an "early poverty axiom" and a "chronic poverty axiom." The early poverty axiom reflects the increasingly well-established phenomenon that poverty early in life is more critical than poverty later in life due to a carry-over impact this can have on an individual's intrinsic wellbeing later in life. The chronic poverty axiom reflects the idea that, for example, two successive spells of poverty of a given intensity are more harmful to an individual's well-being than two spells separated by one or more periods of non-poverty.

We do not presume that our axioms used to reflect the particular pattern or timing of poverty spells within an individual's lifetime are the only reasonable candidates. They do, however, lead to a manageable yet rich way of viewing and measuring lifetime poverty. Certainly one can explore alternative axioms in the same framework as we have developed and we discuss some such possibilities here. A few recent papers have focused on the chronic poverty aspect of lifetime poverty. These include Foster (2007), who explicitly adopts an axiom reflecting time anonymity, and Calvo and Dercon (2007) who explicitly argue against the notion of time-discounting of poverty experiences over an individual's lifetime. ${ }^{12}$ Although we do not propose discounting per se, we do propose an axiom to recognize the heightened implications of poverty early in life. In conjunction with our chronic poverty axiom, this implies a set of weights that is nonincreasing (and possibly decreasing) and concave in time when applied to an individual's lifetime stream of poverty experiences. ${ }^{13}$

The manner in which we capture a concern with chronic poverty is perhaps closest to an axiom used by Bossert, D'Ambrosio and Peragine (2006) to reflect the importance of persistence in the context of measuring social exclusion. They give a higher weight to consecutive periods in a state of social deprivation than to isolated periods. Our axiom is consistent with their notion but extends it to give poverty spells that occur 'closer in time' (but not necessarily contiguously) greater weight than those that occur 'further apart'.

\footnotetext{
${ }^{12}$ See also Cruces (2005), Grab and Grimm (2007) and Carter and Ikegami (2007).

${ }^{13}$ Discounting weights are not consistent once we consider the chronic poverty axiom.
} 
In all of our discussions and derivations except Propositions 2.2 and 2.3, the earlypoverty axiom has been assumed. Recall that this axiom assumes that the poverty weighting function $\alpha(t, T)$ is a nonincreasing function of time $t$. Although we believe this axiom is appealing from the point of view that earlier-life poverty may impact latter-life standards of living but not the other way around, it does not mean that the axiom will be universally accepted. In fact, one may argue that poverty may matter more when the time is getting closer to $T$, end of life. Or one may even suggest that poverty matters more during both ends of life. In the former case, the weighting function will exhibit

$$
\alpha(1, T) \leq \alpha(2, T) \leq \ldots \leq \alpha(T, T)
$$

and for the latter case it is

$$
\alpha(1, T) \geq \alpha(2, T) \geq \ldots \geq a\left(t^{*}-1, T\right) \geq a\left(t^{*}, T\right) \leq a\left(t^{*}+1, T\right) \leq \ldots \leq \alpha(T, T)
$$

for some given period $t^{*}$ in the 'middle'.

For both alternative stipulations of $\alpha(t, T)$, the corresponding dominance conditions can be derived. For all poverty measures satisfying (4.1) and two lifetime consumption profiles $\mathbf{x}$ and $\mathbf{y}, P(\mathbf{x} ; z) \geq P(\mathbf{y} ; z)$ if and only if

$$
\sum_{t=l}^{T} p\left(x_{t}, z\right) \geq \sum_{t=l}^{T} p\left(y_{t}, z\right) \text { for } l=1,2, \ldots, T \text { and } \min \{\bar{x}, z\} \leq \min \{\bar{y}, z\}
$$

the two other conditions for all possible poverty deprivation functions $p()$ can be derived similarly to those of Proposition 2.4. For condition (4.2), a sufficient condition would be

$$
\begin{aligned}
\sum_{t=1}^{l} p\left(x_{t}, z\right) & \geq \sum_{t=1}^{l} p\left(y_{t}, z\right) \text { for } l=1,2, \ldots, t^{*} \\
\sum_{t=l}^{T} p\left(x_{t}, z\right) & \geq \sum_{t=l}^{T} p\left(y_{t}, z\right) \text { for } l=t^{*}, 2, \ldots, T \text { and } \min \{\bar{x}, z\} \leq \min \{\bar{y}, z\} .
\end{aligned}
$$

There are other important issues on societal aggregation of individual poverty deprivations that we leave for further study. These include how to compare individuals who live over different numbers of periods (e.g., overlapping generations), for people who live a different number of years, etc. Also, data on consumption is not always available while data on incomes is. This poses further challenges for implementation of measuring lifetime poverty for any approach, including ours.

In conjunction with other axioms that we believe are compelling, such as a strong monotonicity axiom and an independence axiom, we have developed a number of results characterizing how one can measure lifetime poverty through the use of two components; one being a weighted average of all snapshot poverty levels and another being the poverty of average lifetime consumption (i.e., retrospective poverty). Using 
the PSID data set, we have explored the power of orderings implied by alternative combinations of these axioms based on pairwise comparisons that can be made for any set of well-behaved snapshot poverty indices. Placing no structure on how to compare poverty at different periods of life leads to a very weak general ordering principle. Adding the early poverty axiom and the chronic poverty axiom sequentially markedly improves the power of the ordering procedure. We provide examples of weighting functions that are consistent with both axioms and demonstrate some general properties that such weighting functions must satisfy. We also develop necessary dominance conditions, relating to first and second degree stochastic dominance, that satisfy various combinations of our axioms. We believe these results will be useful in focussing attention on how to properly capture the intent of measurement of lifetime poverty experiences as well as providing insight on how to compare poverty alleviation programs that may have different impacts on atemporal and temporal aspects of poverty. Finally, we considered a path-independence requirement to make the two natural alternative approaches of aggregation consistent. 


\section{References}

Aczél, J. (1966): Lectures on Functional Equations and Their Applications, Academic Press, New York.

Addison, T., D. Hulme, R. Kanbur (2008): Poverty Dynamics: Measurement and Understanding from an Interdisciplinary Perspective, BWPI working paper 19, January.

Atkinson, A. B. and F. Bourguignon (1982): The Comparison of Multi-Dimensional Distributions of Economic Status, Review of Economic Studies XLIX, 183-201.

Baulch, B. and J. Hoddinott (2000): Economic Mobility adn Poverty Dynamics in Developing Countries: Introduction to Special Issue, Journal of Develpment Studies, $36,1-24$.

Bossert, W., C. D'Ambrosio, and V. Peragine (2007): Deprivation and Social Exclusion, Economica, vol. 74, pp. 777-803.

Bourguignon, F. and S. Chakravarty (2002): Multidimensional Poverty Orderings, mimeo, Delta.

Calvo, C. and S. Dercon (2007): Chronic Poverty and All That: The Measurement of Poverty Over Time, Chronic Poverty Research Centre working paper no. 89.

Carter, M. and M. Ikegami (2007): Looking Forward: Theory-based Measures of Chronic Poverty and Vulnerability, Chronic Poverty Research Centre working paper no. 94 .

Chronic Poverty Research Center (2005): The Chronic Poverty Report 2004-05.

Cruces, G. (2005): Income Fluctuations, poverty and Well-Being Over Time: Theory and Application to Argentina, London School of Economics, Discussion paper No. DARP 76.

Dardanoni, V. (1993): Measuring Social Mobility, Journal of Economic Theory 61, 372-394.

Davies, J. and M. Hoy (1994): The Normative Significance of Using Third-Degree Stochastic Dominance in Comparing Income Distributions, Journal of Economic Theory, 64, 520-530.

Davies, J. and M. Hoy (1995): Making Inequality Comparisons When Lorenz Curves Intersect, American Economic Review, 85, 980-86.

Duclos, J.-Y., A. Araar and J. Giles (2006): Chronic and Transient Poverty: Measurement and Estimation, with Evidence from China, IZA discussion paper No. 2078.

Duclos, J.-Y., D. Sahn, and S.D. Younger, Robust Multidimensional Poverty Coomparisons, Economic Journal, 116, 943-968.

Farah, M.J., Shera, D.M., Savage, J.H., Betancourt, L., Giannetta, J.M., Brodsky, N.L., Malmud, E.K. \& Hurt, H. (2006): Childhood poverty: Specific associations with neurocognitive development, Brain Research, 1110, 166-174.

Foster, J. and Shorrocks, A. (1991): Subgroup Consistent Poverty Indices, Econometrica, 59, 687-709.

Foster, J. (2007): A Class of Chronic Poverty Measures, Department of Economics, Vanderbilt University working paper no. 07-W01. 
Grab, J. and M. Grimm (2007): Robust Multiperiod Poverty Comparisons, IberoAmerica Institute for Economic Research working apper no. 160.

Hulme, D. and A. McKay (2005): Identifying and Understanding Chronic Poverty: Beyond Monetary Measures, mimeo, The Chronic Poverty Research Center.

Jalan, J. and M. Ravallion (1998): Determinants of Transient and Chronic Poverty, World Bank Policy Research Paper, working paper no. 1936.

Jalan, J. and M. Ravallion (2000): Is Transient Poverty Different?, Journal of Development Studies, 36, 82-99.

Karcher, T., P. Moyes and A. Trannoy (1995): The Stochastic Dominance Ordering of Income Distribution Over Time: The Discounted Sum of the Expected Utilities of Income, in W. Barnett et al. (Eds.), Social Choice, Welfare and Ethics, Cambridge University Press, Cambridge, pp. 375-408.

Karcher, T., P. Moyes and A. Trannoy (2002): The Stochastic Dominance Ordering of Income Distribution Over Time: The Expected Utility of the Discounted Sum of Income, memio.

Marshall, A. and I. Olkin (1979): Inequalities: Theory of Majorization and Its Applications, New York: Academic Press.

Rodgers, J. and J. Rodgers (1993): Chronic Poverty in the United States, Journal of Human Resources 28 (1), 25-54.

Rudin, W. (1976): Principles of Mathematical Analysis, 3rd Edition, New York: McGraw-Hill.

Saposnik, R. (1981): Rank Dominance in Income Distributions, Public Choice 36: 147-151.

Shorrocks, A. (1980): The Class of Additively Decomposable Inequality Measures, Econometrica 48, 613-625.

Shorrocks, A. and J. Foster (1987): Transfer sensitive Inequality Measures, Review of Economic Studies, 54, 485-97.

Zheng, B. (1997): Aggregate Poverty Measures, Journal of Economic Surveys 11, 123-162.

Zheng, B. (1999) On the Power of Poverty Orderings, Social Choice and Welfare 16, 349-371.

Zheng, B. (2000a): Minimum Distribution-Sensitivity, Poverty Aversion, and Poverty Orderings, Journal of Economic Theory 95, 116-137.

Zheng, B. (2007): Unit-Consistent Poverty Indices, Economic Theory 31, 113-142. 


\section{Appendix}

We describe here in more detail the PSID data set, and our use of it, in generating our empirical illustrations.

\section{Description of the PSID data set}

The data we use is taken from the PSID. This data set is publicly available (see http://psidonline.isr.umich.edu/). We use information only on those individuals who were in the first year of the panel (1967) and for whom we have continued data throughout to year 2002, a total of 670 individuals. For the years 1997, 1999, 2001 no surveys were taken and rather than interpolate values for these years, we ignore them. Collapsing the above noted years of data gives us 33 years of data on each individual. Unfortunately, information on consumption is not available so we use income as a proxy. In each year we know the family size of the unit in which the individual lived and we use this information to generate a sequence of 33 years of income information for each individual. We need comparable incomes across time that take into account changes in the CPI and family size. We also need a consistent poverty line (threshold) for the entire period. We accomplish this by first converting all incomes into real (CPI-adjusted) values, using 1983 as our base year.

The poverty thresholds in 1983 constant dollars vary slightly since they are not based on the all-goods CPI. This variation, however, is small. For a single person household this poverty threshold varies from a minium of $\$ 4,994.91$ to a maximum of $\$ 5,084.69$ in 1983 dollars. To achieve a single poverty line that does not vary by year and is the same for average lifetime income we take the average of the values over the 33 years which is $\$ 5,057.30$. Also for the purpose of consistency, we choose equivalence scales based on the ratio of the poverty line used in the PSID for each family size relative to an individual in a single household. Thus, for example, since the poverty line for a 2-person family is 6487.20 , the equivalence scale to use for a person in a two-person family is $6487.20 / 5057.30=1.28$. Thus, an individual in a family of size 2 is assigned an equivalent individual income equal to the family's income divided by 1.28. Thus, a person is determined to be poor if his equivalized individual income is below the value $\$ 5,057.30$ and this is consistent with the determination of his poverty status according to the family poverty line.

Using time consistent values as described above leads to almost identical annual poverty rates as from the use of year-specific poverty lines used in the PSID. The average annual poverty rate for our subset of individuals is $6.87 \%$ while lifetime poverty (based on average lifetime income) is $3.28 \% .{ }^{14}$ The fraction of those in the population who are never poor (i.e., in any year) is $67.46 \%$. We are interested in

\footnotetext{
${ }^{14}$ That is, based on average lifetime equivalized income, $3.28 \%$ of the individuals in our sample had average income over the 33 years of recorded income below the poverty line of $\$ 5,057.30$ in constant 1983 dollars.
} 
seeing how different criteria (axioms) used to assess lifetime poverty, including those axioms that highlight the importance of chronic and early poverty, lead to different conclusions regarding how many pairs of individuals can be ordered in regard to their lifetime poverty status. Therefore, although we realize our data is not representative of the US population ${ }^{15}$ this is not particularly worrisome since it is only the relative power of the axioms that interest us and this is simply a demonstration of that. In this analysis we concern ourselves only with comparisons based on general classes of poverty indices (i.e., for all deprivation functions $p$ such that $p^{\prime}<0$ or $p^{\prime}<0$ and $\left.p^{\prime \prime}>0\right)$.

Since $67.5 \%$ of the sample never experience poverty it follows that approximately $45.6 \%$ of all possible pairwise comparisons are of no interest since neither individual in such pairs ever experienced poverty. In a further $44 \%$ of pairwise comparisons, one individual is never poor while the other is poor in at least one period of life. Thus, regardless of which axioms one adopts to capture the essence of lifetime and chronic poverty, there is a clear decision about who is more poor. Thus, it is only in about $10 \%$ of all possible comparisons, numbering 23,653 cases, that one has a nontrivial decision to make concerning which individual incurs more lifetime poverty. We focus our attention on these 'interesting cases'.

\section{Implementation}

Implementing the requirements in Propositions 2.2 and 2.3 is straightforward, so we only consider the cases for Propositions 2.4 and 2.5. In Proposition 2.4 we consider the implication of adopting the early poverty axiom that restricts the $\alpha(t, T) \mathrm{s}$ to be nonincreasing in time $t$. The implication for parts (2) and (3) of Proposition 2.4 is that rather than checking for rank dominance and generalized Lorenz dominance, respectively, for the complete vectors $\mathbf{x}$ and $\mathbf{y}$, we must check this for all subvectors of length $1,2, \ldots, \mathrm{T}$. This is to ensure that poverty earlier in life is given prominence in making the ordering as required by the early poverty axiom. To implement comparisons, let $\ell$ represent the first $\ell$ years of a person's life. Then the subvector $\widetilde{\mathbf{x}}^{\ell}=\left(\widetilde{x}_{1}, \widetilde{x}_{2}, \ldots, \widetilde{x}_{\ell}\right)$ provides the censored incomes of the first $\ell$ years, in chronological order. In line with our previous notation, this vector when sorted in ascending order is $\widetilde{\mathbf{x}}^{\ell}=\left(\widetilde{x}_{(1)}, \widetilde{x}_{(2)}, \ldots, \widetilde{x}_{(\ell)}\right)$. To determine if income profile $\mathbf{x}$ displays more lifetime poverty than income profile $\mathbf{y}$ under the early poverty axiom for all deprivation functions $p$ such that $p^{\prime}<0$ one must check if vector $\left(\widetilde{x}_{1}, \widetilde{x}_{2}, \ldots, \widetilde{x}_{\ell}\right)$ rank dominates vector $\left(\widetilde{y}_{1}, \widetilde{y}_{2}, \ldots, \widetilde{y}_{\ell}\right)$ for all $\ell=1, \ldots, T$. This requires $\widetilde{x}_{(t)}^{\ell} \leq \widetilde{y}_{(t)}^{\ell} \forall t=1,2, \ldots, \ell$ AND all $\ell=1,2, \ldots, T$. For the case of all deprivation functions $p$ such that $p^{\prime}<0$ and $p^{\prime \prime}>0$, we require vector $\left(\widetilde{x}_{1}, \widetilde{x}_{2}, \ldots, \widetilde{x}_{\ell}\right)$ generalize Lorenz dominate vector $\left(\widetilde{y}_{1}, \widetilde{y}_{2}, \ldots, \widetilde{y}_{\ell}\right)$ for all $\ell=1, \ldots, T$. This requires checking $\sum_{t=1}^{k} \tilde{x}_{(t)} \leq \sum_{t=1}^{k} \tilde{y}_{(t)}$ for $k=1,2, \ldots, \ell$ AND all $\ell=1,2, \ldots, T$. As in all cases one must also check that $\min \{\bar{x}, z\} \leq \min \{\bar{y}, z\}$.

\footnotetext{
${ }^{15}$ Perhaps the most important reason our sample is not representative is that we only choose individuals who have lived throughout the 33 year period and were traced and agreed to be interviewed in each of those years.
} 
To implement results (2) and (3) of Proposition 2.5 we first need to construct the following vectors $x_{l}$, as defined below, and then check for rank dominance (case 2 ) and generalized Lorenz dominance (case 3) based on the sorted vectors $x_{l}$ (in nondescending order):

$$
\tilde{\mathbf{x}}_{l}=(\underbrace{\tilde{x}_{1}, . ., \tilde{x}_{1}}_{l \text { times }}, \ldots, \underbrace{\tilde{x}_{T-l+1}, \ldots, \tilde{x}_{T-l+1}}_{l \text { times }}, \underbrace{\tilde{x}_{T-l+2}, . ., \tilde{x}_{T-l+2}}_{l-1 \text { times }}, \underbrace{\tilde{x}_{T-l+3}, . ., \tilde{x}_{T-l+3}}_{l-2 \text { times }}, \ldots, \underbrace{\tilde{x}_{T}}_{1 \text { time }})
$$

Thus, for $l=1$ we get just the entire vector.

$$
\tilde{\mathbf{x}}_{1}=\left(\widetilde{x}_{1}, \widetilde{x}_{2}, \ldots, \widetilde{x}_{T}\right)
$$

For $l=2$ we get:

$$
\tilde{\mathbf{x}}_{2}=(\underbrace{\tilde{x}_{1}, \tilde{x}_{1}}_{l=2 \text { times }}, \underbrace{\tilde{x}_{2}, \tilde{x}_{2}}_{l=2 \text { times }}, \ldots, \underbrace{\tilde{x}_{T-1}, \tilde{x}_{T-1}}_{l=2 \text { times }}, \underbrace{\tilde{x}_{T}}_{l-1=1 \text { times }})
$$

For $l=3$ we get:

$$
\tilde{\mathbf{x}}_{l}=(\underbrace{\tilde{x}_{1}, \tilde{x}_{1}, \tilde{x}_{1}}_{l=3 \text { times }}, \underbrace{\tilde{x}_{2}, \tilde{x}_{2}, \tilde{x}_{2}}_{l=3 \text { times }}, \ldots, \underbrace{\tilde{x}_{T-2}, \tilde{x}_{T-2}, \tilde{x}_{T-2}}_{l=3 \text { times }}, \underbrace{\tilde{x}_{T-1}, \tilde{x}_{T-1}}_{l-1=2 \text { times }}, \underbrace{\tilde{x}_{T}}_{l-2=1 \text { times }})
$$

et cetera.

When the last step, $l=T$, is reached, $\widetilde{x}_{1}$, appears $T$ times, and then successive elements appear $T-1, T-2, \ldots$, times, with $\widetilde{x}_{T}$ appearing once.

The following table summarizes the results of the empirical illustrations of the propositions.

Rankings between those who experience poverty (23,653 cases)

\begin{tabular}{ccc} 
Proposition & Number ranked & Percent ranked \\
\hline Proposition 2.2 & 2,118 & $9 \%$ \\
Proposition 2.3 (part 2) & 14,885 & $63 \%$ \\
Proposition 2.3 (part 3) & 17,983 & $73 \%$ \\
Proposition 2.4 (part 2) & 8,936 & $38 \%$ \\
Proposition 2.4 (part 3) & 9,914 & $42 \%$ \\
Proposition 2.5 (part 2) & 10,913 & $46 \%$ \\
Proposition 2.5 (part 3) & 14,859 & $63 \%$
\end{tabular}

